\title{
A Machine-Checked Direct Proof of the Steiner-Lehmus Theorem
}

\author{
Ariel Kellison \\ Department of Computer Science, Cornell University \\ Ithaca, NY, USA \\ ak2485@cornell.edu
}

\begin{abstract}
A direct proof of the Steiner-Lehmus theorem has eluded geometers for over 170 years. The challenge has been that a proof is only considered direct if it does not rely on reductio ad absurdum. Thus, any proof that claims to be direct must show, going back to the axioms, that all of the auxiliary theorems used are also proved directly. In this paper, we give a proof of the Steiner-Lehmus theorem that is guaranteed to be direct. The evidence for this claim is derived from our methodology: we have formalized a constructive axiom set for Euclidean plane geometry in a proof assistant that implements a constructive logic and have built the proof of the Steiner-Lehmus theorem on this constructive foundation.

CCS Concepts: • Theory of computation $\rightarrow$ Constructive mathematics; Logic and verification.
\end{abstract}

Keywords: constructive logic, proof assistants, constructive geometry, foundations of mathematics

\section{ACM Reference Format:}

Ariel Kellison. 2022. A Machine-Checked Direct Proof of the SteinerLehmus Theorem. In Proceedings of the 11th ACM SIGPLAN International Conference on Certified Programs and Proofs (CPP '22), January 17-18, 2022, Philadelphia, PA, USA. ACM, New York, NY, USA, 9 pages. https://doi.org/10.1145/3497775.3503682

\section{Introduction}

The Steiner-Lehmus theorem, which states that if two internal angle bisectors of a triangle are equal then the triangle is isosceles, was posed by C. L. Lehmus in 1840 . Since the publication of Jakob Steiner's 1844 proof of the theorem, it has become somewhat infamous for the many failed attempts of a direct proof; that is, one that does not use reductio ad absurdum. Numerous allegedly direct proofs have appeared over the years, only to later be discredited due to their reliance on reductio ad absurdum by means of auxiliary theorems with

Publication rights licensed to ACM. ACM acknowledges that this contribution was authored or co-authored by an employee, contractor or affiliate of the United States government. As such, the Government retains a nonexclusive, royalty-free right to publish or reproduce this article, or to allow others to do so, for Government purposes only.

CPP '22, January 17-18, 2022, Philadelphia, PA, USA

(c) 2022 Copyright held by the owner/author(s). Publication rights licensed to ACM.

ACM ISBN 978-1-4503-9182-5/22/01_..\$15.00

https://doi.org/10.1145/3497775.3503682

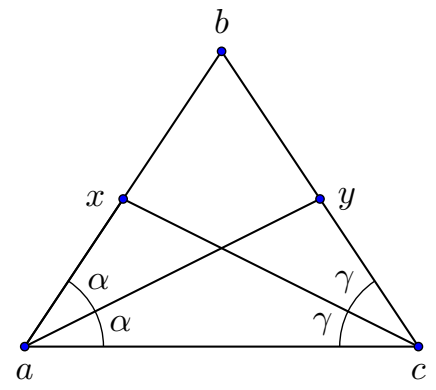

Figure 1. The Steiner-Lehmus theorem: if $a y \cong c x, \angle c a y \cong$ $\angle y a b$, and $\angle b c x \cong x c a$ then $a b \cong c b$.

indirect proofs. Given the many failures to provide a direct proof, attempts have been made to prove that a direct proof can't possibly exist $[6,14]$. Recently, we have been reassured that a direct proof does exist [13], but we have yet to see one. Thus, the history of the Steiner-Lehmus theorem serves as 177 years of evidence that a human can't account for all instances of the use of particular rule of logic, even in the proof of a theorem that many would consider to be rather elementary.

In this paper, we provide a direct proof of the SteinerLehmus theorem. Our guarantee of directness is obtained on modern terms: by formalizing a constructive axiom set for Euclidean plane geometry in the Nuprl proof assistant [5] and building a proof of the Steiner-Lehmus theorem on this constructive foundation.

Our direct proof of the Steiner-Lehmus theorem is given in Section 5 and can be found in the Nuprl library ${ }^{1}$. Of the many indirect proofs published since the theorem was first posed by Lehmus in 1840, ours is superficially the most similar to the proof given by R.W. Hogg in 1982 [10]. Like many of the former proofs of the Steiner-Lehmus theorem, the proof given by Hogg uses reductio ad absurdum both explicitly and implicitly; the implicit use is hidden in auxiliary constructions. In comparison, our proof is completely free from the use of reductio ad absurdum. The atomic relations, axioms, and definitions used in our direct proof of the Steiner-Lehmus theorem are described in Sections 3-4. The

\footnotetext{
${ }^{1}$ The entire formalization of geometry can be found at http: //www.nuprl.org/LibrarySnapshots/Published/Version2/Mathematics/ euclidean!plane!geometry/index.html and the Steiner-Lehmus theorem can be found at http://www.nuprl.org/LibrarySnapshots/Published/Version2/ Mathematics/euclidean!plane!geometry/Steiner-LehmusTheorem.html.
} 
soundness of our axioms with respect to the constructive reals provides additional assurance that the axioms themselves do not harbor any hidden instances of reductio ad absurdum. The basis for our model in the constructive reals is given in Section 7.

Before introducing the necessary axioms and definitions in Sections 3-4, we first provide background on the methods of constructive logic that clarify how a direct proof of the Steiner-Lehmus theorem was obtained.

\section{Constructive Proof, Stability, and Decidability}

When the Steiner-Lehmus theorem was first posed in 1840, the field of logic was not fully formed. Perhaps if it had been, and constructive logic had flourished, geometers would have realized that the Steiner-Lehmus theorem is an example of a proof of negation. In particular, the notion of a triangle being isosceles is constructively understood to be a negative statement about a strict notion of inequality of segment lengths. While it is generally assumed that the use of case distinctions is rejected in constructive reasoning, the proof of a negation is an instance where reasoning by cases is constructively valid.

Proof by contradiction (reductio ad absurdum) is a classically admissible reasoning principle that allows one to prove a proposition $P$ by assuming $\neg P$ and deriving absurdity. A proof of negation is superficially similar, as one provides proof of the proposition $\neg P$ by assuming $P$ and deriving absurdity. The validity of the two is clearly differentiated in constructive reasoning by the general rejection of the law of double negation elimination for arbitrary propositions $P$ : in constructive logic, a proof of negation remains perfectly valid, while a proof by reductio ad absurdum, which is classically equivalent to the law of double negation elimination, does not.

While the law of double negation elimination is not constructively valid for arbitrary propositions $P$, it is provably true for some propositions. Propositions for which double negation elimination is provably true are referred to as stable:

Definition 2.1. (Stability)

A proposition $P$ is stable if $\neg \neg P \rightarrow P$ holds.

In constructive logic, all negative propositions are stable. Specifically,

Theorem 2.2. For all propositions $P, \neg \neg(\neg P) \rightarrow \neg P$.

Furthermore, the proof of a stable proposition permits case distinctions. In particular, the double negation of the law of excluded middle for arbitrary propositions is constructively valid:

Theorem 2.3. For all propositions $P, \neg \neg(P \vee \neg P)$.
Thus, in the proof of a stable proposition $P$, one need only introduce the double negation of the desired case distinction as a hypothesis and apply the appropriate elimination rules to obtain the desired cases.

Finally, a strictly stronger notion than stability is decidability:

Definition 2.4. (Decidability)

A proposition $P$ is decidable if $P \vee \neg P$ holds.

While the decidability of arbitrary propositions does not hold constructively, it is a provable property for many propositions $P$, specifically those for which there is an algorithm for deciding which of $P$ or $\neg P$ holds.

\subsection{Stable Relations in Constructive Geometry}

We do not take the stability or decidability of any of our atomic or defined relations as axioms. This choice clearly distinguishes our theory from those that take the stability of equality, betweenness, or congruence as axioms $[1,2,11]$. Instead, we define equivalence, collinearity, betweenness, and congruence (Definitions 3.5, 3.6, 3.7, and 3.10) negatively in terms of a strictly positive atomic relation. It follows from Theorem 2.2 that these are stable relations. Our axioms therefore clearly distinguish the geometric propositions that constructively permit case distinctions from those that do not.

Finally, the choice to not take the stability of equality as an axiom is driven by the desired model, which is the Nuprl implementation of the constructive reals. If we were to take the stability of equality as an axiom, then equality and equivalence would coincide, which does not hold in the Nuprl implementation of the constructive reals [3, p. 3].

\section{Constructive Geometric Primitives and Relations}

Our axioms rely on two atomic relations on points: a quaternary relation representing an ordering on segment lengths and a ternary relation for plane orientation. These relations are introduced using the formalism of type theory to parallel their implementation in the Nuprl proof assistant. For example, the statement $a$ : Point is to be read as " $a$ of type Point."

\subsection{Segments in Type Theory}

The segment type is defined as the Cartesian product of two points. The elements of a Cartesian product are pairs, denoted $\langle a, b\rangle$. If $a$ has type Point and $b$ has type Point, then $\langle a, b\rangle$ has type Point $\times$ Point:

$$
\frac{a: \text { Point } \quad b: \text { Point }}{\langle a, b\rangle: \text { Point } \times \text { Point }}
$$


We will abbreviate segment pairs $\langle a, b\rangle$ by simply writing $a b$ :

$$
\frac{a: \text { Point } \quad b: \text { Point }}{a b: \text { Segment }}
$$

When it is necessary to decompose the points constituting a segment $a b$, we may write fst $(a b)$ and snd( $a b)$ for $a$ and $b$ respectively.

\subsection{Atomic Relations and Apartness}

Constructive geometry traditionally utilizes a binary apartness relation in place of equality $[9,11,15,16]$. A notable exception is the axiom set presented by Lombard and Vesley [12], which uses an atomic six place relation and defines a binary apartness relation in terms of the atomic six place relation. In this work, we use an atomic quaternary strictly greater than relation to define a binary apartness relation. In particular, given the four points $a, b, c$, and $d$, if the length of the segment $a b$ is strictly greater than the length of the segment $c d$, then the atomic ordering relation on points will be denoted by $a b>c d$. A binary apartness relation on points can then be defined using the atomic strictly greater than relation as follows.

Definition 3.1 (Apartness of points). The points $a$ and $b$ satisfy an apartness relation if the length of the segment $a b$ is strictly greater than the length of the null segment $a a$ :

$$
a \# b:=a b>a a \text {. }
$$

The strictly greater than relation is used to define two additional quaternary relations on points: apartness of segment lengths and a non-strict ordering of segment lengths.

Definition 3.2 (Apartness of segment lengths). The length of the segments $a b$ and $c d$ satisfy a length apartness relation if either the length of the segment $a b$ is strictly greater than the length of the segment $c d$ or the length of the segment $c d$ is strictly greater than the length of the segment $a b$ :

$$
a b \# c d:=a b>c d \vee c d>a b .
$$

Definition 3.3 (Non-strict order of segment lengths). The length of $a b$ is greater than or equal to the length of $c d$ if the length of $c d$ is not strictly greater than the length of $a b$ :

$$
a b \geq c d:=\neg c d>a b .
$$

The atomic relation for plane orientation used in this work is adopted from the constructive axiom set for Euclidean plane geometry introduced in [11]: given the three points $a, b$, and $c$, if the point $a$ lies to the left of the segment $b c$, then the atomic leftness relation on points will be denoted by $\operatorname{Left}(a, b c)$. We use the atomic leftness relation to define an apartness relation between a point a segment as follows.
Definition 3.4 (Apartness of a point and a segment). The point $a$ lies apart from the segment $b c$ if it is either to the left of the segment $b c$ or to the left of the segment $c b$ :

$$
a \# b c:=\operatorname{Left}(a, b c) \vee \operatorname{Left}(a, c b) .
$$

\subsection{The Constructive Interpretation of Classical Geometric Relations}

The classical relations of equivalence, collinearity, betweenness, and congruence are defined using the atomic relations of leftness and strictly greater than. In this section, we give the definitions of these relations, and provide the proof of a useful theorem as a simple example of proving stable propositions using constructive logic.

Definition 3.5 (Equivalence on points). The points $a$ and $b$ are equivalent if they do not satisfy the binary apartness relation on points (Definition 3.1):

$$
a \equiv b:=\neg a \# b .
$$

As is mentioned in Section 2.1, equivalence and equality do not coincide: while equality on points implies equivalence, equivalence does not imply equality.

Definition 3.6 (Collinearity). The points $a, b$, and $c$ are collinear if they do not satisfy the apartness relation between a point and a segment:

$$
\operatorname{Col}(a b c):=\neg(a \# b c) .
$$

Definition 3.7 (Betweenness). The point $b$ lies between the points $a$ and $c$ if $a, b$, and $c$ are collinear and the length of the segment $a c$ is not strictly greater than the lengths of $a b$ and $b c$ :

$$
B(a b c):=\operatorname{Col}(a b c) \wedge a c \geq a b \wedge a c \geq b c .
$$

Note that the above definition coincides with what is referred to as non-strict betweenness. That is, the points $a, b$, and $c$ may be equivalent.

Theorem 3.8 (Collinear Cases). Any three collinear points satisfy a weak betweenness relation.

$$
\begin{aligned}
& \forall a, b, c: \text { Point. } \operatorname{Col}(a b c) \Rightarrow \\
& \neg \neg(B(a b c) \vee B(c a b) \vee B(b c a) \vee a \equiv b \vee a \equiv c \vee b \equiv c) .
\end{aligned}
$$

Proof. The stability of the conclusion allows for reasoning by cases on

$$
\neg \neg(a \# b \vee \neg a \# b),
$$


and similarly for $a \# c$ and $b \# c$. Consider the case where $a \# b$, $a \# c$, and $b \# c$. Assume

$\neg(B(a b c) \vee B(c a b) \vee B(b c a) \vee a \equiv b \vee a \equiv c \vee b \equiv c)$, and prove false. Observe that $\neg B(a b c) \wedge \neg B(c a b) \wedge \neg B(b c a)$ follows from the assumption. From $\neg B(a b c)$ it follows that

$$
\neg \neg(a \# b c \vee a b>a c \vee b c>a c) \text {. }
$$

Stability of the conclusion allows for elimination of the double negation for each betweenness relation, and expanding the disjunctions results in absurdity.

Definition 3.9 (Strict Betweenness). The point $b$ lies strictly between the points $a$ and $c$ if the point $b$ lies between the points $a$ and $c$, and the points $a, b$, and $c$ satisfy apartness relations:

$$
S B(a b c):=B(a b c) \wedge a \# b \wedge b \# c .
$$

Definition 3.10 (Congruence). The segments $a b$ and $c d$ are congruent if they do not satisfy the apartness relation on segment lengths (Definition 3.2):

$$
a b \cong c d:=\neg a b \# c d .
$$

Definition 3.11 (Out). The point $p$ lies out along the segment $a b$ if it is separated from both $a$ and $b$ and satisfies some weak betweenness relation with $a$ and $b$. Observe that this definition can be viewed as using an constructive interpretation of the classical disjunction used in Definition 6.1 of [17].

$$
\operatorname{out}(p, a b):=p \# a \wedge p \# b \wedge \neg(\neg B(p a b) \wedge \neg B(p b a))
$$

The universally quantified axioms introduced in Section 4 imply that collinearity, betweenness, and congruence are equivalence relations.

\subsection{Angle Relations}

Our proof of the Steiner-Lehmus theorem required constructive definitions for angle congruence, the sum of two angles, and angle ordering. The following definition of angle congruence is taken from Tarski [17], but has been modified to use the appropriate constructive relations.

Definition 3.12 (Congruent Angles). The angles $a b c$ and $x y z$ are congruent if the segments of each angle are distinct and there exist points making the corresponding segments of the two angles congruent:

$$
\begin{aligned}
& a b c \cong a x y z:= \\
& a \# b \wedge b \# c \wedge x \# y \wedge y \# z \wedge \\
& \left(\exists a^{\prime}, c^{\prime}, x^{\prime}, z^{\prime}: \text { Point. } B\left(b a a^{\prime}\right) \wedge B\left(b c c^{\prime}\right) \wedge B\left(y x x^{\prime}\right) \wedge\right. \\
& \left.B\left(y z z^{\prime}\right) \wedge b a^{\prime} \cong y x^{\prime} \wedge b c^{\prime} \cong y z^{\prime} \wedge a^{\prime} c^{\prime} \cong x^{\prime} z^{\prime}\right) .
\end{aligned}
$$

The set of Axioms U, introduced in Section 4, imply that angle congruence is an equivalence relation.

Definition 3.13 (Sum of two angles).

$$
\begin{aligned}
& a b c+x y z=\operatorname{def}:= \\
& \quad \exists p, p^{\prime}, d^{\prime}, f^{\prime}: \text { Point } . a b c \cong_{a} \operatorname{dep} \wedge f e p \cong_{a} x y z \wedge \\
& B\left(e p^{\prime} p\right) \wedge \text { out }\left(e d d^{\prime}\right) \wedge \text { out }\left(e f f^{\prime}\right) \wedge S B\left(d^{\prime} p^{\prime} f^{\prime}\right)
\end{aligned}
$$

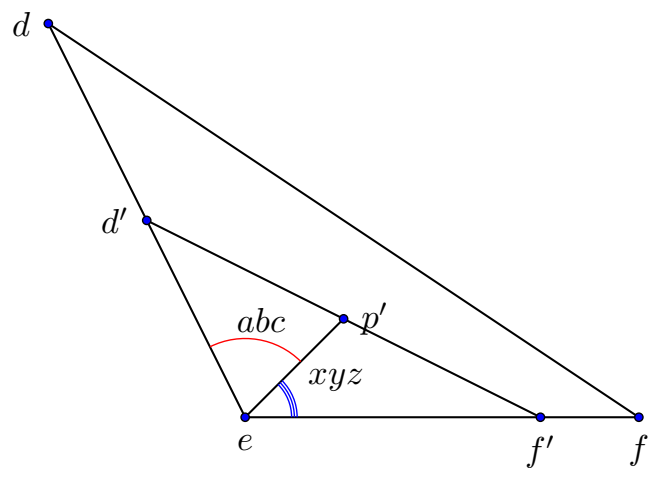

Figure 2. A diagram of Definition 3.13: $a b c+x y z=d e f$ with $p^{\prime}=p$.

Definition 3.14 (Angle Inequality).

$$
\begin{aligned}
& a b c<<_{a} x y z=: \neg \text { out }(y x z) \wedge \\
& \exists p, p^{\prime}, x^{\prime}, z^{\prime}: \text { Point } . a b c \cong_{a} x y p \wedge \\
& B\left(y p^{\prime} p\right) \wedge \text { out }\left(y x x^{\prime}\right) \wedge \text { out }\left(y z z^{\prime}\right) \wedge \\
& \neg B(x y p) \wedge B\left(x^{\prime} p^{\prime} z\right)^{\prime} \wedge p^{\prime} \# z^{\prime}
\end{aligned}
$$

Our axioms imply that angle inequality is a transitive relation for angles satisfying the ternary apartness relation on points (Definition 3.4).

\subsection{Parallel Segments}

The following definition of parallel segments was essential to our proof of the Steiner-Lehmus theorem.

Definition 3.15 (Parallel Segments). The segments $a b$ and $c d$ are parallel if $a \# b$ and $c \# d$ and there do not exist points $x$ and $y$ collinear with $a b$ such that $x$ and $y$ lie on opposite sides of $c d$ :

$$
\begin{gathered}
a b \| c d:=a \# b \wedge c \# d \wedge \neg(\exists x, y: \text { Point. } \operatorname{Col}(x a b) \wedge \\
\operatorname{Col}(y a b) \wedge \operatorname{Left}(x, c d) \wedge \operatorname{Left}(y, d c)) .
\end{gathered}
$$

According to our axioms introduced in the following section, parallelism is a symmetric and reflexive relation but not a transitive relation. Transitivity of parallelism is known to be equivalent to the parallel postulate [4], which is not an axiom of the theory presented in this paper. 


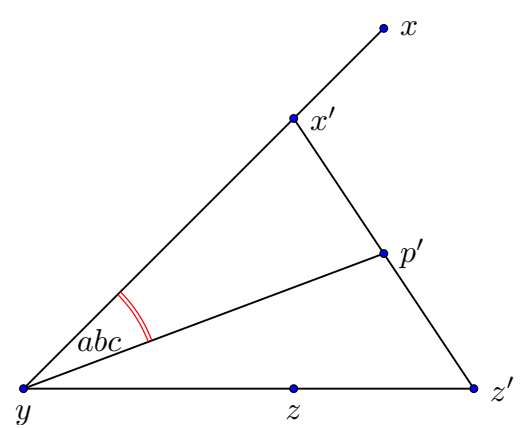

(a) Definition 3.14 for typical angles, with $p=p^{\prime}$.

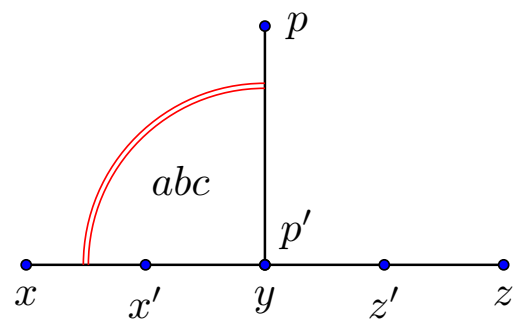

(b) Definition 3.14 for a straight angle.

Figure 3. Definition 3.14, $a b c<{ }_{a} x y z$.

\section{Construction Postulates and Axioms}

The axioms are introduced here in two separate groups: Axioms $\mathrm{U}$ and Axioms $\mathrm{C}$. Axioms $\mathrm{U}$ are universally quantified and contain no disjunctions or existential quantifiers. The application of any one of these axioms does not result in a geometric construction. Axioms $\mathrm{C}$ are constructor axioms relying on disjunctions and existential quantifiers. As a result, the axioms in group $\mathrm{C}$ have a convenient functional reading which may be used in proofs.

From the Axioms listed below, Axioms U7-U13 and Axioms $\mathrm{C} 1-\mathrm{C} 3$ are also used in a previous set of constructive axioms for Euclidean geometry [11]. Unlike the previous system, the relations of betweenness, congruence, and apartness are not primitives of our theory, they are instead defined as described in Section 3.3. Furthermore, as previously noted, the stability of congruence and betweenness are not taken as axioms in the current work. Finally, the axioms C4 and C5 have been simplified to remove the assertion of the existence of redundant points.

\subsection{Universally Quantified Axioms}

Axiom U1. $\forall a, b, c$ : Point. $b c \geq a a$

Axiom U2. $\forall a, b, c, d$ : Point $. a b>c d \Rightarrow a b \geq c d$

Axiom U3. $\forall a, b, c$ : Point. $b a>a c \Rightarrow b \# c$

Axiom U4.

$\forall a, b, c, d, e, f:$ Point $. a b>c d \Rightarrow c d \geq e f \Rightarrow a b>e f$
Axiom U5.

$\forall a, b, c, d, e, f:$ Point $. a b \geq c d \Rightarrow c d>e f \Rightarrow a b>$ ef

Axiom U6. $\forall a, b, c$ : Point. $B(a b c) \Rightarrow b \# c \Rightarrow a c>a b$

Axiom U7. $\forall a, b, c$ : Point. $\operatorname{Left}(a, b c) \Rightarrow \operatorname{Left}(b, c a)$

Axiom U8. $\forall a, b, c$ : Point. $\operatorname{Left}(a, b c) \Rightarrow b \# c$

Axiom U9. $\forall a, b, c, d$ : Point.$B(a b d) \Rightarrow B(b c d) \Rightarrow B(a b c)$

We take an constructive versions of Tarski's Five-Segment axiom and Upper Dimension axiom [17].

Axiom U10 (Five-Segment).

$$
\begin{array}{r}
\forall a, b, c, d, w, x, y, z: \text { Point. }(a \# b \wedge B(a b c) \wedge B(w x y) \wedge \\
a b \cong w x \wedge b c \cong x y \wedge a d \cong w z \wedge b d \cong x z) \Rightarrow \\
c d \cong y z
\end{array}
$$

Axiom U11 (Upper Dimension).

$$
\begin{array}{r}
\forall a, b, c, x, y: \text { Point . } a x \cong a y \Rightarrow b x \cong b y \Rightarrow \\
c x \cong c y \Rightarrow x \# y \Rightarrow \operatorname{Col}(a b c)
\end{array}
$$

Axiom U12 (Convexity of Leftness).

$\forall a, b, x, y, z:$ Point. $\operatorname{Left}(x, a b) \wedge \operatorname{Left}(y, a b) \wedge B(x z y) \Rightarrow$

$$
\operatorname{Left}(z, a b)
$$

\section{Axiom U13.}

$\forall a, b, c, y:$ Point.$a \# b c \Rightarrow y \# b \Rightarrow \operatorname{Col}(y a b) \Rightarrow y \# b c$

\subsection{Construction Postulates}

Axiom C1 (Cotransitivity of separated points:).

$\forall a, b, c:$ Point. $a \# b \Rightarrow a \# c \vee b \# c$

Axiom C2 (Plane Separation). If the points $u$ and $v$ lie on opposite sides of the segment $a b$, then the point $x$, collinear with $a b$, exists between $u$ and $v$.

$$
\begin{array}{r}
\forall a, b, u, v: \text { Point. }(\operatorname{Left}(u, a b) \wedge \operatorname{Left}(v, b a) \Rightarrow \\
\exists x: \text { Point. } \operatorname{Col}(a b x) \wedge B(u x v))
\end{array}
$$

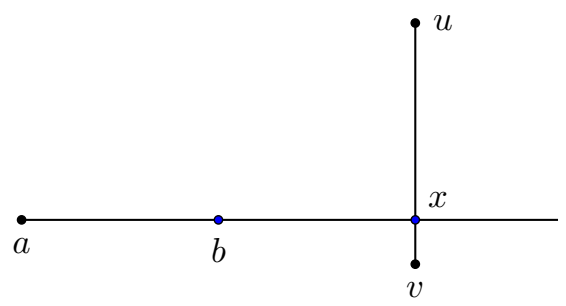

Figure 4. Axiom C2: plane separation. 
Axiom C3 (Non-triviality).

$$
\exists a, b \text { : Point. } a \# b
$$

Axiom C4 (Straightedge-Compass). The straight-edge compass axiom constructs a single point of intersection between a circle and a segment (see Figure 5):

$$
\begin{array}{r}
\forall a, b, c, d \text { : Point . }(a \# b \wedge B(c b d)) \Rightarrow \\
\exists u \text { : Point . } c u \cong c d \wedge B(a b u) \wedge(b \# d \Rightarrow b \# u)
\end{array}
$$

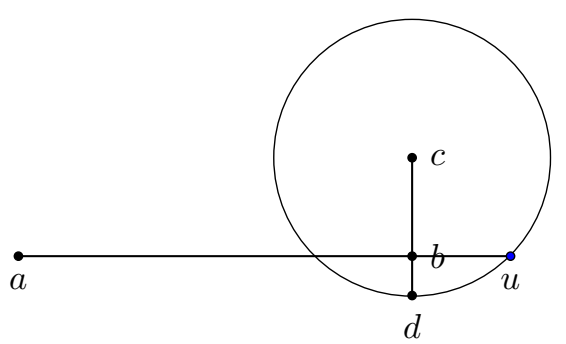

Figure 5. Axiom $\mathrm{C} 4$, the straight-edge compass construction.

Axiom C5 (Compass-Compass). The compass-compass axioms constructs a single point of intersection between two circles (see Figure 6):

$$
\forall a, b, c, d: \text { Point. } a \# c \wedge
$$

$(\exists p, q$ : Point $. a b \cong a p \wedge c d>c p \wedge c d \cong c q \wedge a b>a q) \Rightarrow$ $\exists u$ : Point. $a b \cong a u \wedge c d \cong c u \wedge \operatorname{Left}(u, a c)$

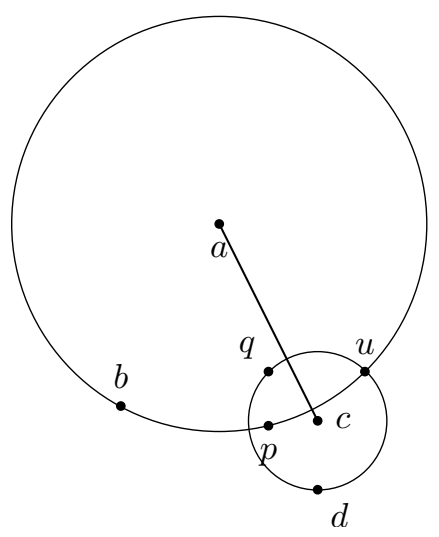

Figure 6. Axiom C5, the compass-compass construction.

\section{The Steiner-Lehmus Theorem}

The conclusion of the Steiner-Lehmus theorem is stable and so it suffices to prove the double negation of auxiliary theorems with constructive content. Thus, rather than proving a lemma stating that from two points along the sides of any triangle, a parallelogram can be constructed such that one side of the parallelogram lies along one side of the triangle, we prove the following lemma:

\section{Lemma 5.1.}

$\forall a, b, c, x, y$ : Point. $(a \# b c \wedge S B(a x b) \wedge S B(c y b) \Rightarrow$

$$
\begin{array}{r}
\neg \neg(\exists t \text { : Point. } y t\|a x \wedge x t\| a y \wedge \\
a x \cong y t \wedge x t \cong a y \wedge t \# b c) .
\end{array}
$$

Proof. Construct the midpoint $m$ along the segment $x y$ using Euclid I.10 (Theorem 6.5), and extend the segment am to construct the point $t$ such that $a m \cong m t$ by Lemma 6.1. Now, the angle congruence $x m a \cong_{a} y m t$ follows from Euclid I.15 (Theorem 6.6), and the congruence relations $a x \cong y t$ and $x t \cong a y$ follow from Euclid I.4 (Theorem 6.2) and Axiom U10, respectively. The angle congruence $a x y \cong_{a} t y x$ then follows by definition, and from Euclid I.27 (Theorem 6.9) it follows that $a x \| y t$ and $x t \|$ ay. Finally, stability of the conclusion allows for reasoning by cases on $t \# b c$ or $\operatorname{Col}(t b c)$.

If $\mathrm{Col}(t b c)$ then by Lemma 6.10 the point $t$ must be the point $p$ such that $S B(b p c)$ and $S B(a m p) ; p$ is guaranteed to exist by construction using Lemma 6.14. Without loss of generality, from $a \# b c$ assume $\operatorname{Left}(a, c b)$. From Lemma 6.11, it follows that Left $(c, x a)$. Now, construct the point $q$ by Lemma 6.1 such that $S B(c b q)$ and $S B(y p q)$. It follows from Lemma 6.13 that $\operatorname{Left}(q$, ax $)$, contradicting $a x \| y t$.

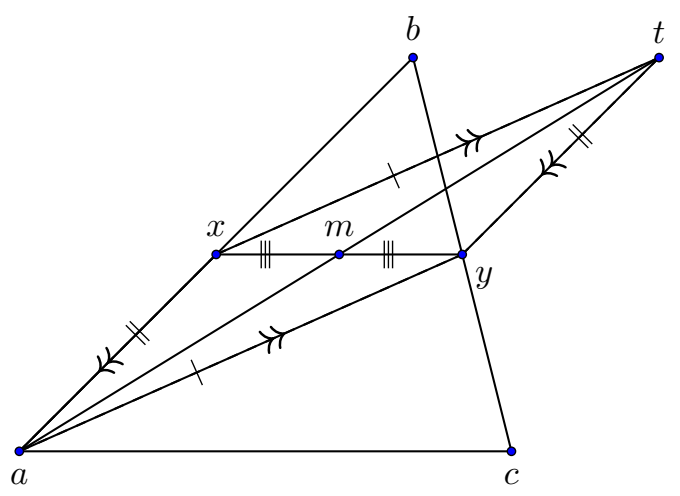

Figure 7. Lemma 5.1

Theorem 5.2 (Steiner-Lehmus).

$$
\begin{aligned}
& \forall a, b, c, x, y \text { : Point. }(a \# b c \wedge S B(a x b) \wedge S B(c y b) \wedge \\
& \left.a y \cong c x \wedge x a y \cong_{a} c a y \wedge y c x \cong_{a} a c x \Rightarrow a b \cong c b\right) .
\end{aligned}
$$

Proof. Construct the parallelogram ayxt by Lemma 5.1. From Euclid I.5 (Theorem 6.3) it follows that $x c t \cong_{a} x t c$. The angle sum relations $x t y+y t c \cong_{a} x t c$ and $x c y+y c t \cong_{a} x c t$ follow by definition from construction of the point $q$ using 
Axiom C2 such that $S B(q y c), B(t y y), S B(x q t)$, and $B(c q q)$. Now, stability of the conclusion allows for reasoning by cases on $c y>a x$ or $\neg(c y>a x)$.

If $c y>a x$, then $c y>y t$ by definition of the parallelogram ayxt. From Euclid I.25 (Theorem 6.8) it follows that $a c x<<_{a} c a y$, and therefore $x c y<<_{a} x t y$. It then follows from Euclid I.18 (Theorem 6.7) that $t c y<_{a} y t c$, which, along with Lemma 6.15 and the angle sum relations $x t y+y t c \cong_{a} x t c$ and $x c y+y c t \cong_{a} x c t$, yields the contradiction $x t y<_{a} x c y$.

If $\neg(c y>a x)$, it follows that $\neg \neg(a x>c y \vee a x \cong c y)$ : stability of the conclusion allows for elimination of the double negation, so that we can reason by cases on $a x>c y$ or $a x \cong c y$. A contradiction is reached for $a x>c y$ by the same reasoning used for $c y>a x$.

Finally, if $a x \cong c y$, it follows from Definition 3.12 that $x a c \cong_{a} y c a$. Theorem 6.4 then yields $a b \cong c b$, as desired.

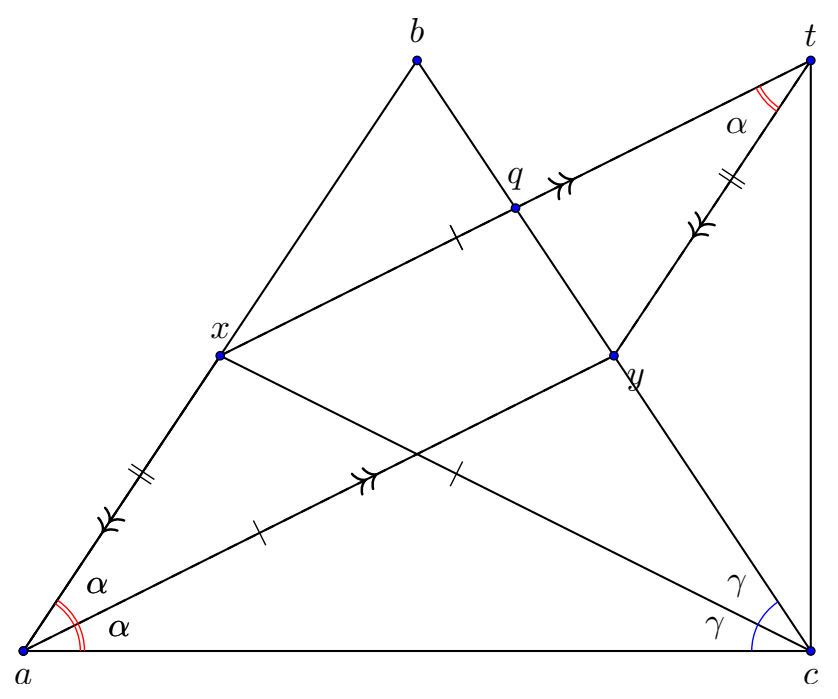

Figure 8. The Steiner-Lehmus Theorem 5.2

\section{Essential Auxiliary Theorems}

This section contains only the statements of the auxiliary theorems used in the proof of the Steiner-Lehmus theorem (Theorem 5.2) and Lemma 5.1. The names given to the theorems in this section match their names in the Nuprl library ${ }^{2}$. Some definitions used in the Nuprl statement of a theorem may occur unfolded in the following theorem statements for clarity.

Theorem 6.1 (geo-extend-exists).

$$
\forall q, a, b, c: \text { Point. } q \# a \Rightarrow \exists x \text { : Point. } B(q a x) \wedge a x \cong b c .
$$

\footnotetext{
${ }^{2}$ http://www.nuprl.org/LibrarySnapshots/Published/Version2/ Mathematics/euclidean!plane!geometry/index.html
}

Theorem 6.2 (Euclid-Prop4). If two triangles have two sides equal to two sides respectively, and have the angles contained by the equal straight lines equal, then they also have the base equal to the base, the triangle equals the triangle, and the remaining angles equal the remaining angles respectively, namely those opposite the equal sides.

$$
\begin{aligned}
& \forall a, b, c, x, y, z: \text { Point } . a \# b \wedge a \# c \wedge b \# c \wedge x \# y \wedge x \# z \wedge \\
& y \# z \wedge a b \cong x y \wedge b c \cong y z \wedge a b c \cong_{a} x y z \Rightarrow \\
& a c \cong x z \wedge b a c \cong a y x z \wedge b c a \cong_{a} y z x .
\end{aligned}
$$

Theorem 6.3 (Euclid-Prop5). In isosceles triangles the angles at the base equal one another, and, if the equal straight lines are produced further, then the angles under the base equal one another.

$$
\begin{gathered}
\forall a, b, c, x, y: \text { Point } . a b \cong a c \wedge a \# b c \wedge S B(a b x) \wedge \\
S B(a c y) \Rightarrow a b c \cong_{a} a c b \wedge x b c \cong_{a} y c b .
\end{gathered}
$$

Theorem 6.4 (Euclid-Prop6). If in a triangle two angles equal one another, then the sides opposite the equal angles also equal one another.

$$
\forall a, b, c: \text { Point. } c \# a b \Rightarrow c a b \cong{ }_{a} c b a \Rightarrow c a \cong c b .
$$

Theorem 6.5 (Euclid-Prop10). To bisect a given straight line.

$$
\begin{array}{r}
\forall a, b \text { : Point } . a \# b \Rightarrow \\
\exists d \text { : Point. } S B(a d b) \wedge a d \cong d b .
\end{array}
$$

Theorem 6.6 (vert-angles-congruent). If two straight lines cut one another, then they make the vertical angles equal to one another.

$$
\forall a, b, c, x, y \text { : Point. } S B(a b x) \wedge S B(c b y) \Rightarrow a b c \cong_{a} x b y .
$$

Theorem 6.7 (Euclid-Prop18). In any triangle the angle opposite the greater side is greater.

$$
\forall a, b, c \text { : Point. } a \# b c \wedge a c>a b \Rightarrow b c a<_{a} a b c .
$$

Theorem 6.8 (Euclid-Prop25). If two triangles have two sides equal to two sides respectively, but have the base greater than the base, then they also have the one of the angles contained by the equal straight lines greater than the other.

$$
\begin{gathered}
\forall a, b, c, d, e, f: \text { Point. } a \# b c \wedge d \# e f \wedge a b \cong d e \wedge \\
a c \cong d f \wedge b c>e f \Rightarrow e d f<<_{a} b a c .
\end{gathered}
$$

In the following theorem, the Left relation is used in the antecedent to capture the notion of "alternate angles." 
Theorem 6.9 (Euclid-Prop27). If a straight line falling on two straight lines makes the alternate angles equal to one another, then the straight lines are parallel to one another:

$$
\begin{array}{r}
\forall a, b, c, d, x, y: \text { Point. }(\operatorname{Col}(x a b) \wedge \operatorname{Col}(y c d) \wedge a \# b \wedge \\
\left.c \# d \wedge \operatorname{Left}(a, y x) \wedge \operatorname{Left}(c, x y) \wedge a x y \cong_{a} c y x\right) \Rightarrow \\
a b \| c d .
\end{array}
$$

Lemma 6.10 (geo-intersection-unicity).

$$
\begin{array}{r}
\forall a, b, c, d, p, q: \text { Point } . \neg \operatorname{Col}(a b c) \wedge c \# d \wedge \\
\operatorname{Col}(a b p) \wedge \operatorname{Col}(a b q) \wedge \operatorname{Col}(c d p) \wedge \operatorname{Col}(c d q) \Rightarrow p \equiv q .
\end{array}
$$

Lemma 6.11 (left-convex). Given a segment $a b$ and $a$ point $x$ lying to the left of it, the point $y$ lying out from $x$ that along the segment ax or bx is in the same half-plane as $x$.

$\forall a, b, x, y:$ Point. $\operatorname{Left}(x, a b) \wedge($ out $(a x y) \vee$ out $(b x y)) \Rightarrow$

$$
\operatorname{Left}(y, a b)
$$

Lemma 6.12 (geo-left-out). Given a segment $a b$ and $a$ point $c$ lying out from $b$ along $a b$, if the point $x$ lies to the left of $a b$, then $x$ also lies to the left of ac.

$$
\forall a, b, c, x: \text { Point. } \operatorname{Left}(x, a b) \wedge \operatorname{out}(a b c) \Rightarrow \operatorname{Left}(x, a c)
$$

Lemma 6.13 (strict-between-left-right).

$$
\begin{array}{r}
\forall a, b, c, x, y: \text { Point. } \operatorname{Left}(x, a b) \wedge \operatorname{Col}(a b c) \wedge S B(x c y) \Rightarrow \\
\operatorname{Left}(y, b a)
\end{array}
$$

Theorem 6.14 (outer-pasch-strict).

$$
\begin{gathered}
\forall a, b, c, x, q \text { : Point. } x \# b q \wedge S B(b q c) \wedge S B(q x a) \Rightarrow \\
\exists p \text { : Point. } S B(b x p) \wedge S B(c p a) .
\end{gathered}
$$

Lemma 6.15 (hp-angle-sum-lt4). If the sum of the strict angles $a b c$ and $x y z$ is equal to the sum of the strict angles $a^{\prime} b^{\prime} c^{\prime}$ and $x^{\prime} y^{\prime} z^{\prime}$, and $x^{\prime} y^{\prime} z^{\prime}$ is less than $x y z$, then it must be the case that the angle abc is less than the angle $a^{\prime} b^{\prime} c^{\prime}$.

$$
\begin{array}{r}
\forall a, b, c, x, y, z, i, j, k: \text { Point } . \\
\forall a^{\prime}, b^{\prime}, c^{\prime}, x^{\prime}, y^{\prime}, z^{\prime}, i^{\prime}, j^{\prime}, k^{\prime}: \text { Point } . \\
a b c+x y z \cong i j k \wedge a^{\prime} b^{\prime} c^{\prime}+x^{\prime} y^{\prime} z^{\prime} \cong i^{\prime} j^{\prime} k^{\prime} \wedge \\
i j k \cong{ }_{a} i^{\prime} j^{\prime} k^{\prime} \wedge a^{\prime} \# b^{\prime} c^{\prime} \wedge x^{\prime} \# y^{\prime} z^{\prime} \wedge x \# y z \wedge i \# j k \wedge \\
x^{\prime} y^{\prime} z^{\prime}<x y z \Rightarrow a b c<a^{\prime} b^{\prime} c^{\prime} .
\end{array}
$$

\section{A Model on the Constructive Reals}

The soundness of our axioms with respect to the Nuprl implementation of the constructive reals [3] is implied by the following interpretations of our primitives ${ }^{3}$.

Definition 7.1. If $x \in \mathbb{R}$ is the length of the segment $a b$ and $y \in \mathbb{R}$ is the length of the segment $c d, x$ is strictly greater than $y$ if and only if there exists a natural number $n$ such that the $n$th rational terms of $x$ and $y$ differ by more than four:

$$
x>_{\mathbb{R}} y:=\exists n \in \mathbb{N} . x(n)>_{\mathbb{Q}} y(n)+4 .
$$

Note that the ordering relation $>_{\mathbb{Q}}$ on the rational numbers is decidable (Definition 2.4) while the ordering relation $>_{\mathbb{R}}$ on the constructive reals is not.

Definition 7.2. Given the real coordinates $\left(x_{0}, y_{0}, 1\right)$,

$\left(x_{1}, y_{1}, 1\right),\left(x_{2}, y_{2}, 1\right)$ of the points $a, b$ and $c$, respectively, the point $a$ lies left of the segment $b c$ if and only if the determinant of the matrix formed by the points $a, b$ and $c$ is strictly positive:

$$
\operatorname{Left}(a, b c):=\left|\begin{array}{lll}
x_{0} & y_{0} & 1 \\
x_{1} & y_{1} & 1 \\
x_{2} & y_{2} & 1
\end{array}\right|>_{\mathbb{R}} 0
$$

The soundness of our axioms with respect to the constructive reals provides additional assurance that our axioms do not use reductio ad absurdum. Although the axioms presented in this paper differ (as described in Section 4) from those presented in [11], only minor modifications were necessary for the soundness proofs.

Finally, while the constructive real model for our axioms guarantees that a direct proof of the Steiner-Lehmus theorem exists in the constructive reals, it says nothing about the existence of direct proof in the classical reals.

\section{Conclusion}

We have introduced here for the first time a proof of the Steiner-Lehmus theorem that is entirely absent of the use of reductio ad absurdum and can therefore be considered fully direct. This theorem was proved in the constructive logic of the Nuprl proof assistant using a novel axiomatization of Euclidean plane geometry without the parallel postulate. The crux of the proof is the realization that congruence in constructive geometry is a stable relation, and that the proof of a stable relation permits double negation elimination and therefore also case distinctions.

Finally, we conclude by addressing the suggestion that the many years of failed attempts to find a direct proof of

\footnotetext{
${ }^{3}$ The proofs of soundness for the Axiom sets $\mathrm{U}$ and $\mathrm{C}$ can be found at http://www.nuprl.org/LibrarySnapshots/Published/Version2/ Mathematics/reals!model!euclidean!geometry as the theorems r2-basic-geo-axioms and r2-eu_wf, respectively.
} 
the Steiner-Lehmus theorem was cause to celebrate the indispensability of reductio ad absurdum. In particular, a discussion of the Steiner-Lehmus theorem given in a geometry textbook by Coxeter and Greitzer [7] includes the popular quote of G. H. Hardy [8]: Reductio ad absurdum, which Euclid loved so much, is one of a mathematician's finest weapons. We instead propose the following:

Double negation is one of a mathematician's finest weapons, and a proof assistant one of her most steadfast companions.

\section{Acknowledgements}

The author would like to thank Robert Constable and Mark Bickford for being patient teachers of intuitionism and type theory, and would also like to acknowledge their contributions to the development of constructive Euclidean plane geometry in Nuprl. The author would also like to thank Andrew Appel for his helpful feedback and encouragement. Finally, the author would like to thank the anonymous reviewers whose comments helped to improve this paper.

The author acknowledges that this material is based upon work supported by the U.S. Department of Energy, Office of Science, Office of Advanced Scientific Computing Research, Department of Energy Computational Science Graduate Fellowship under Award Number DE-SC0021110. This report was prepared as an account of work sponsored by an agency of the United States Government. Neither the United States Government nor any agency thereof, nor any of their employees, makes any warranty, express or implied, or assumes any legal liability or responsibility for the accuracy, completeness, or usefulness of any information, apparatus, product, or process disclosed, or represents that its use would not infringe privately owned rights. Reference herein to any specific commercial product, process, or service by trade name, trademark, manufacturer, or otherwise does not necessarily constitute or imply its endorsement, recommendation, or favoring by the United States Government or any agency thereof. The views and opinions of authors expressed herein do not necessarily state or reflect those of the United States Government or any agency thereof.

\section{References}

[1] Michael Beeson. 2015. A Constructive Version of Tarski's Geometry. Annals of Pure and Applied Logic 166, 11 (2015), 1199-1273. https: //doi.org/10.1016/j.apal.2015.07.006

[2] Michael Beeson. 2017. Brouwer and Euclid. Indagationes Mathematicae 29 (05 2017). https://doi.org/10.1016/j.indag.2017.06.002

[3] Mark Bickford. 2016. Constructive Analysis and Experimental Mathematics using the Nuprl Proof Assistant. http://www.nuprl.org/ documents/Bickford/reals.pdf.

[4] Pierre Boutry, Charly Gries, Julien Narboux, and Pascal Schreck. 2019. Parallel Postulates and Continuity Axioms: A Mechanized Study in Intuitionistic Logic Using Coq. Fournal of Automated Reasoning 62 (2019), 1 - 68. https://doi.org/10.1007/s10817-017-9422-8

[5] R. L. Constable, S. F. Allen, H. M. Bromley, W. R. Cleaveland, J. F. Cremer, R. W. Harper, D. J. Howe, T. B. Knoblock, N. P. Mendler, P. Panangaden, J. T. Sasaki, and S. F. Smith. 1986. Implementing Mathematics with the Nuprl Proof Development System. Prentice-Hall, Inc., USA.

[6] John Conway and Alex Ryba. 2014. The Steiner-Lehmus AngleBisector Theorem. The Mathematical Gazette 98, 542 (2014), 193 203.

[7] H. S. M. Coxeter and S. L. Greitzer. 1967. Geometry Revisited (1 ed.). Vol. 19. Mathematical Association of America.

[8] G.H. Hardy. 1940. A Mathematician's Apology. Cambridge University Press (1940).

[9] A. Heyting. 1959. Axioms for Intuitionistic Plane Affine Geometry. Studies in Logic and the Foundations of Mathematics 27 (1959), 160 173. https://doi.org/10.1016/S0049-237X(09)70026-6 The Axiomatic Method.

[10] R. W. Hogg. 1982. Equal Bisectors Revisited. The Mathematical Gazette 66, 438 (1982), 304 - 304. https://doi.org/10.2307/3615522

[11] Ariel Kellison, Mark Bickford, and Robert Constable. 2019. Implementing Euclid's Straightedge and Compass Constructions in Type Theory. Annals of Mathematics and Artificial Intelligence 85 (2019), 175 - 192. https://doi.org/10.1007/s10472-018-9603-0

[12] Melinda Lombard and Richard Vesley. 1998. A Common Axiom Set for Classical and Intuitionistic Plane Geometry. Annals of Pure and Applied Logic 95, 1 (1998), 229-255. https://doi.org/10.1016/S01680072(98)00017-7

[13] Victor Pambuccian. 2018. Negation-Free and Contradiction-Free Proof of the Steiner-Lehmus Theorem. Notre Dame fournal of Formal Logic 59, 1 (2018), 75 - 90. https://doi.org/10.1215/00294527-2017-0019

[14] J. J. Sylvester. 1852. On a Simple Geometrical Problem Illustrating a Conjectured Principle in the Theory of Geometrical Method. Philos. Mag. 4 (1852), 366-369.

[15] Dirk van Dalen. 1996. 'Outside' as a Primitive Notion in Constructive Projective Geometry. Geometriae Dedicata 60, 1 (1996), 107 - 111. https://doi.org/10.1007/BF00150870

[16] Jan von Plato. 2003. Proofs and Types in Constructive Geometry (Tutorial). Technical Report. Rome, Italy.

[17] Alfred Tarski Wolfram Schwabhäuser, Wanda Szmielew. 1983. Metamathematische Methoden in der Geometrie. Springer-Verlag, Berlin, Heidelberg. 